\title{
Catheter Fixation Behind the Urachus: A Novel Laparoscopic Technique to Revise Malfunctioning Peritoneal Dialysis Catheters
}

\author{
Ahmad Kachoei ${ }^{1}$, Maryam Rafiei ${ }^{2}$, Enayatollah Noori ${ }^{3}$, Sarvenaz Salahi ${ }^{4}$ and Mohammad Mahdi Shater ${ }^{3^{*}}$ \\ ${ }^{1}$ Department of Surgery, Shahid Beheshti Hospital, Qom University of Medical Sciences, Qom, Iran \\ ${ }^{2}$ Clinical Research Department Center, Shahid Beheshti Hospital, Qom University of Medical Sciences, Qom, Iran \\ ${ }^{3}$ Student Research Committee, Medical School, Qom University of Medical Sciences, Qom, Iran \\ ${ }^{4}$ Clinical Research Department Center, Rasool-E-Akram Hospital, Iran University of Medical Sciences, Tehran, Iran
}

"Corresponding author: Mohammad Mahdi Shater, Student Research Committee, Medical School, Qom University of Medical Sciences, Qom, Iran, Tel: 982536623175; Fax: 982536122855; E-mail: dr.shater@chmail.ir

Received date: October 31, 2018; Accepted date: November 13, 2018; Published date: November 20, 2018

Copyright: () 2018 Kachoei A, et al. This is an open-access article distributed under the terms of the Creative Commons Attribution License, which permits unrestricted use, distribution and reproduction in any medium, provided the original author and source are credited.

\begin{abstract}
Peritoneal dialysis is one of the types of renal replacement therapy which is commonly used in patients with the end-stage renal disease. The cost-effectiveness and easy usage are the advantages of peritoneal dialysis. However, peritoneal dialysis is not always free of a problem and among all of its complications, catheter tip migration and omental wrapping are more common as are considered as the causes of catheter malfunctioning. Different techniques are used to correct the malfunctioning catheters such as manipulator techniques, laparoscopic repositioning with tip suturing, omentectomy and omentopexy. In this article, we are proposing two cases, which had been referred by peritoneal catheter malfunctioning and then received a new method of surgery successfully and didn't have any complications after 36 months follow up. This method could simplify the procedure of operation and urachus ligament and reduce time and cost of the process.
\end{abstract}

Keywords: Laparoscopy; Peritoneal dialysis catheter; Urachus

\section{Introduction}

Peritoneal dialysis is preferred as the first choice of dialysis modality for pediatric and adult patients with the end-stage renal disease. This modality represents more several advantages than hemodialysis such as greater survival for the first two years, cost-effectiveness and preservation of residual renal function [1,2]. However, peritoneal dialysis is not free of problems. Some of its complications can make failures in function of the catheter including catheter mal-positioning, catheter migration and omental wrap around the catheter [3,4]. Catheter tip migration and omental wrapping are the most frequent laparoscopic finding in a patient who investigated for their malfunctioning catheter $[5,6]$.

Type of the technique of catheter insertion (laparoscopy versus open surgery) affects the incidence of catheter-related complications and survival of catheter; laparoscopic insertion technique provides a higher survival and less migration [1]. Current clinical practice guidelines indicated that there is not any proof to confirm the association between the type of catheter and the rate of its malfunctioning $[7,8]$.

There are different techniques that are used to repair the malfunctioning catheter such as manipulator modalities (e.g. wire and laparoscopic manipulation), laparoscopic repositioning with tip suturing, omentectomy, and omentopexy $[6,7]$ we described a new laparoscopic technique that can resolve the malfunctioning catheter due to migration and omental wrapping.

\section{Case 1}

F.Q was a 13 years old female with deafness, diminished visual acuity and mental retardation who was presented with ESRD due to a heredity renal disease. In order to initiation of peritoneal dialysis; as an alternative therapy; a double cuff, coiled, swan neck, Tenchkhoff catheter was placed in the pelvic region for the first time within an open surgery method. The anesthetic modality was performed based on the default procedure by Drager device.

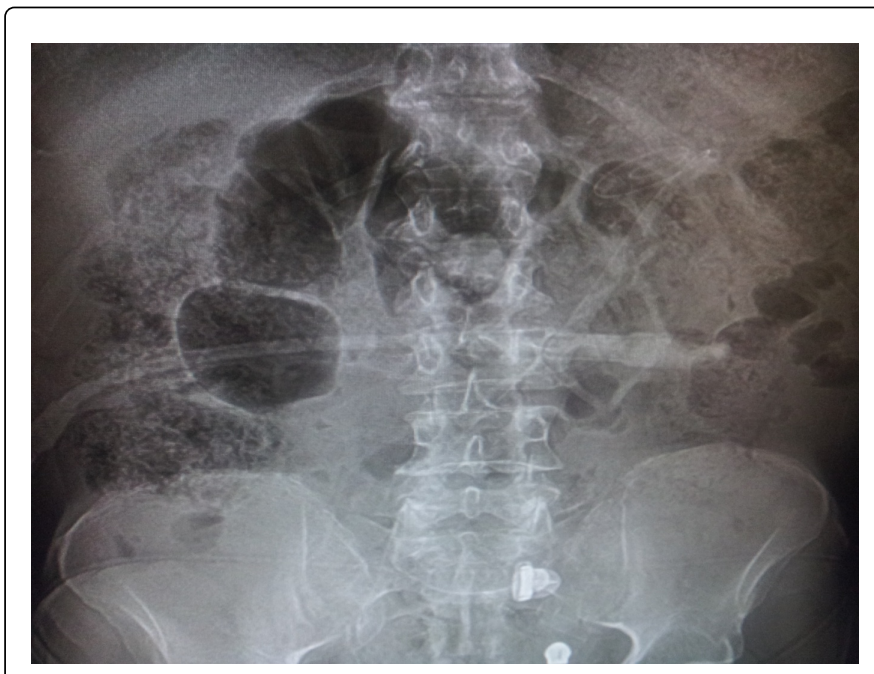

Figure 1: Catheter migrated to the left upper quadrant of the abdomen. 
After 98 days of the primary catheter insertion, the patient represented with complains of catheter failure characterized by poor dialysate flow in both directions and consequently the signs of volume overload. Catheter migration, a common cause of catheter malfunction, was suspected as the first probable cause of catheter failure in this patient and confirmed by an upright abdominal radiography that showed catheter tip position in epigastrium (Figure $1)$.

\section{Surgical Technique}

The patient underwent a laparoscopic surgery for catheter revision, under general anesthesia based on default method and by the device of Drager. The abdominal wall was incised at 3 points; first $1 \mathrm{~cm}$ below the left costal margin where a $10 \mathrm{~mm}$ trocar was inserted for laparoscope passing, and two another; right and left lower quadrant for two $5 \mathrm{~mm}$ trocar insertion. The peritoneal cavity was investigated by laparoscope. After establishing, pneumoperitoneum, omental wrapping, and catheter tip position in epigastrium were detected and no adhesion band was found.

By locating two trocars that were placed on lower quadrants, the migrated catheter was taken and led to pelvic cavity, then catheter passed behind urachus (median umbilical ligament) and placed in the space between the bladder and anterior pelvic wall (Figure 2). To confirm the optimum function, the catheter was irrigated by normal saline and heparin and good flow in both directions were indicated. Trocars were removed then fascia and subcutaneous tissue was closed by several interrupted 4-0 Vicryl suture and skin was repaired by the horizontal mattress 4-0 nylon sutures. Surgery, lasted 15 minutes, from the initiation of the incising the abdominal wall until the last incision site got closed.

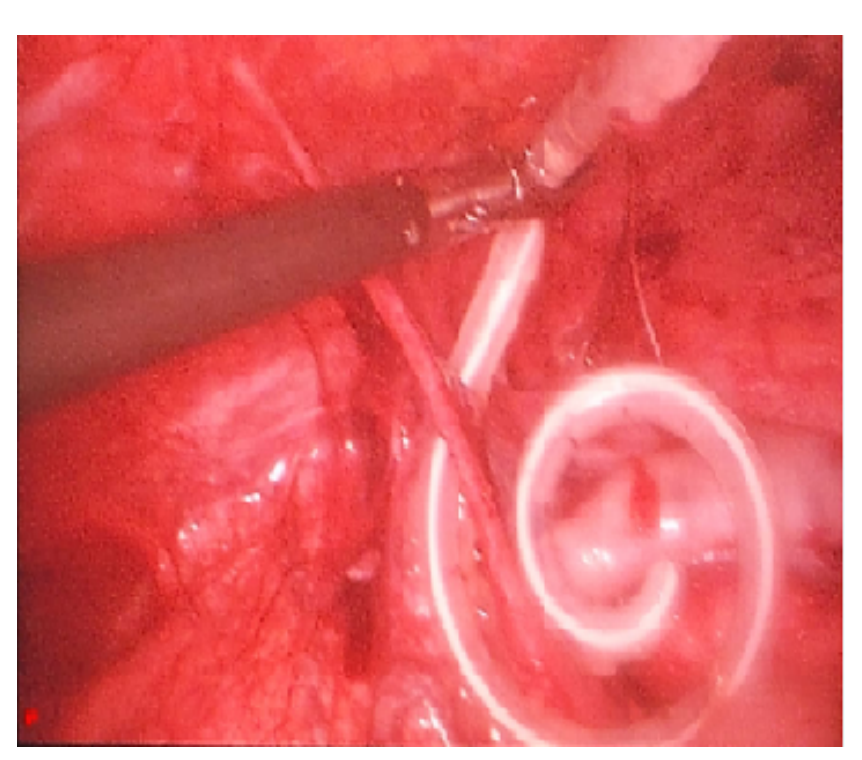

Figure 2: Catheter passed behind urachus.

Peritoneal dialysis began one week after revision and catheter was irrigated by normal saline and heparin during the first week after the operation. During the period of the 36 months follow up, no correlated complications and recurrences were observed.

\section{Case 2}

Z.G was a 73 years old male with ESRD in the background of diabetic nephropathy and presented with alteration in mental status and volume overload. Laboratory studies revealed hemoglobin $=10$ $\mathrm{mg} / \mathrm{dl}$, hematocrits $=32 / 2$, FBS $=258 \mathrm{mg} / \mathrm{dl}$, blood urine nitrogen $(\mathrm{BUN})=192 \mathrm{mg} / \mathrm{dl}$ and $\mathrm{Cr}=7 / 1 \mathrm{mg} / \mathrm{dl}$. After 2 hours of hemodialysis to improve patient's clinical status and providing a better condition for operation, Patient underwent an open surgery in order to place a double cuff, coiled, swan neck, Tenchkoff catheter for the beginning of peritoneal dialysis.

Two months after initial catheter placement, the patient represented with a complaint of poor bidirectional dialysate flow. Catheter malposition was confirmed by an abdominal radiography that showed the placement of catheter's tip-in right upper quadrant.

\section{Surgical Technique}

The patient underwent laparoscopic revision of malfunctioning catheter. Under general anesthesia based on the default method and by Drager device, 3 points of abdominal wall were incised by open insertion of trocars technique. A $10 \mathrm{~mm}$ trocar inserted through an incision that located $1 \mathrm{~cm}$ below the left costal margin for laparoscope passing. Two trocars (each was $5 \mathrm{~mm}$ ) were inserted via the other incisions in left and right lower quadrants to perform catheter repositioning procedure. Laparoscopic peritoneal observation indicated the pneumoperitoneum, omental wrapping, and of catheters tip in right upper quadrant and no adhesion band. By meaning of a grasper, the migrated catheter was led to the pelvic cavity and passed behind the median umbilical ligament and fixated by this natural available anatomic structure in a place between pelvic wall and bladder.

In order to an examination of catheter function, catheter irrigated with normal saline and heparin that indicated good bidirectional dialysate flow. The trocars were removed and to avoid any leakage, fascia and subcutaneous tissues were sutured by permanent interrupted Vicryl suture and skin was repaired by horizontal mattress 4-0 nylon suture. The procedure lasted 17 minutes. Peritoneal dialysis began after one week of catheter irrigation with normal saline and heparin. In a course of 36 months of follow up, no complications and no recurrent episodes of malfunctioning were detected.

\section{Discussion}

In this modality, the abdominal wall was incised with an open insertion technique of the trocar at 3 points: $1 \mathrm{~cm}$ below the left costal margin, right and left lower quadrant. This technique was performed wisely by surgeons to reduce the chance of accidental visceral injury. The determined trocars insertion sites provided the best visualization of both peritoneal and pelvic cavity and facilitate performing every maneuver of repositioning procedure and give better access to pelvic structures.

The urachus is an embryonic remnant from involution of the Allantois and the ventral cloaca which is attaching the bladder dome to the umbilicus $[9,10]$. Using this anatomic structure as an anchoring agent for peritoneal dialysis catheter fixation in the pelvic cavity where the best filtration achieved, affords numerous benefits. 
This technique eliminates the demand of suturing the catheter to the abdominal wall, which is a difficult procedure that requires more time and depends on surgeon proficiency and experience. Fixing the catheter in the pelvic region within the space between the bladder and pelvic wall, of where the greater omentum does not exist, reduces the probability of omental wrapping in the future. Hence, this procedure can reduce the rate of complications without the need for further change in the normal structure of peritoneal cavities such as omentectomy and omentopexy.

Stiff wire manipulation with or without fluoroscopic guidance is a technique for malfunctioning catheter revision. In one study by Kappel et al. 118 manipulations ( 70 cases for the first time and 48 cases for the second time), due to the malposition, fibrin clot, or kinked catheter, were conducted [11]. A successful manipulation was defined as a functional peritoneal dialysis catheter for a period of 30 days after manipulation. There was an overall success rate of $64 \%-67 \%$ for initial manipulations and $48 \%$ for repeated manipulations [11]. This technique accompanied by recurrent episodes of malfunctioning and required further interventions (48 from 70 ).

Wire manipulation techniques, despite their simple performance and the low rate of associated complications, can't resolve some of the problems in the peritoneal cavity such as the adhesion bands formation which can cause probable catheter malfunction.

Although these problems are eliminated by laparoscopic manipulator techniques, recurrent episodes remain as the most important problem with these modalities because the catheter is not fixed through these procedures.

Laparotomy and laparoscopic correction with or without omentectomy and catheter fixation are another technique for revision of the catheter malposition [12-14].

In one study by Lee et al., thirteen patients with nonfunctioning peritoneal dialysis catheters underwent 16 laparoscopic procedures. In all patients, regardless of the cause of the malfunctioning, laparoscopic omentectomy and catheter fixation to the anterior pelvic wall was performed. One-ten $\mathrm{mm}$ and two-five $\mathrm{mm}$ trocars were utilized. Eight catheters remained functional following omentectomy without further intervention. Five patients (38\%) experienced recurrent catheter malfunctioning [14]. Through our procedure, the structure of momentum did not change and the demand of omentectomy was eliminated.

Jwo et al. used a mini-laparotomy approach for salvage and catheter fixation in their study. In 11 patients, a $2 \mathrm{~cm}$ incision was used at $5 \mathrm{~cm}$ caudally to the previous peritoneal entrysite to correct the malfunctioning catheter, with the concomitant fixation of the catheter to the peritoneum. The mean operating time was 41 minutes (range: 35-56 minutes). Although laparoscopic surgery often lasts more than laparotomy [15] in our laparoscopic technique, both operations lasted about 15-17 minutes that can be described as a superiority to other laparoscopic or laparotomy revision modalities and provided the permanent catheter function by using of the catheter fixation.

This technique can potentially decrease the rate of catheter malfunctioning as well as the need for further catheter revisions and the morbidity rate related to recurrent episodes.

\section{Conclusion}

In order to the novel modality, the malfunctioned migrated catheter was led to the pelvic cavity and passed through urachus (median umbilical ligament) and placed in the space between the bladder and the pelvic wall. Consequently, the catheter fixed by urachus naturally and started functioning. This technique could simplify the procedure of surgery by and urachus ligament as a natural anatomic structure and reduce the time and the cost of the procedure. It could be considered as an alternative procedure.

\section{Conflict of Interests}

The authors report no conflict of interests.

\section{References}

1. Hagen SM, Lafranca JA, Steyerberg EW, IJzermans JNM, Dor FJMF (2013) Laparoscopic versus open peritoneal dialysis catheter insertion: A meta-analysis. PLoS ONE 8: 1-11.

2. Mendes ML, Alves CA, Bucuvic EM, Dias DB, Ponce D (2017) Peritoneal dialysis as the first dialysis treatment option initially unplanned. J Bras Nefrol 39: 441-446.

3. Singh N, Davidson I, Minhajuddin A, Gieser S, Nurenberg M, et al (2010) Risk factors associated with peritoneal dialysis catheter survival: A nine-year single center study in 315 patients. J Vasc Access 11: 316-322.

4. Fujiwara M, Soda T, Okada T, Kanamaru H, Inoue T, et al. (2017) Bowel perforation by a peritoneal dialysis catheter: Report of two cases. BMC Nephrol 18: 312 .

5. Yilmazlar T, Kirdak T, Bilgin S, Yavuz M, Yurtkuran M (2006) Laparoscopic findings of peritoneal dialysis catheter malfunction and management outcomes. Peri Dial Int 26: 374-379.

6. Goh HY (2008) Omental folding: A novel laparoscopic technique for salvaging peritoneal dialysis catheters. Perit Dial Int 28: 626-631.

7. Cox TC, Blair LJ, Huntington CR, Prasad T, Kercher KW, et al. (2016) Laparoscopic versus open peritoneal dialysis catheter placement. Surg Endosc 30: 899-905.

8. Figueiredo A, Leong Goh B, Jenkins S, W Johnson D, Mactier R, et al. (2010) Clinical practice guidelines for peritoneal access. Perit Dial Int 30: 424-429.

9. Villavicencio CP, Adam SZ, Nikolaidis P, Yaghmai V, Miller FH (2016) Imaging of the urachus: Anomalies, complications, and mimics. Radiographics 36: 2049-2063.

10. Cappele O, Sibert L, Descargues J, Delmas V, Grise P (2001) A study of the anatomic features of the duct of the urachus. Surg Radiol Anat 23: 229-235.

11. Kappel JE, Ferguson GM, Kudel RM, Kudel TA, Lawlor BJ, et al. (1995) Stiff wire manipulation of peritoneal dialysis catheters. Adv Perit Dial 11: 202-207.

12. Li JR, Cheng CH, Chiu KY, Cheng CL, Yang CR, et al. (2013) Minilaparotomy salvage of malfunctioning catheters in peritoneal dialysis. Perit Dial Int 33: 46-50.

13. Song JH, Lee KJ, Lee SW, Kim MJ (2001) Incisional hernia after corrective omentectomy for peritoneal dialysis catheter malposition. Adv Perit Dial 17: 132-137.

14. Lee M, Donovan JF (2002) Laparoscopic omentectomy for salvage of peritoneal dialysis catheters. J Endourol 16: 241-244.

15. Jwo SC, Chen KS, Lee CC, Chen HY (2010) Prospective randomized study for comparison of open surgery with laparoscopic-assisted placement of Tenckhoff peritoneal dialysis catheter-a single-center experience and literature review. J Surg Res 159: 489-496. 